\title{
Adaptive Patient Education Framework Featuring Personalized Cardiovascular Risk Management Interventions
}

\author{
Selena Davis and Syed Sibte Raza Abidi \\ Health Informatics Laboratory, Faculty of Computer Science, Dalhousie University, \\ Halifax, NS, Canada, B3H 1W5 \{sdavis, sraza\}@cs.dal.ca
}

\begin{abstract}
The PULSE project objectives are to generate and evaluate a webbased personalized educational intervention for the management of cardiovascular risk. We present a web-based adaptive hypermedia system to create and deliver the personalized education material to the patient. The adaptive personalization framework is based on a patient profile created by combining an electronic patient data capture template, the Systematic COronary Risk Evaluation (SCORE) algorithm, and a Stage of behaviour Change determination model. The interventions are designed to address both medical and psychosocial aspects of risk management and, as such, we combine staged lifestyle modification materials and non-staged messages based on Canadian clinical guidelines to motivate personal risk management.
\end{abstract}

\section{Introduction}

Patient education, especially for chronic health conditions, is becoming increasingly complex because the educational content needs to conform to the longitudinal healthcare needs of the patient. Computer based patient education allows for the delivery of educational content to the patient; lately the emergence of health webportals allows for the dissemination of generic healthcare information [1]. However, patients prefer healthcare information that is personalized to their individual needs and situation [2], and evidence shows that personalized information is more likely to be read, remembered, experienced as personally relevant and in turn has a greater impact in motivating patients to make a behaviour change [3]. Adaptive hypermedia systems offer the functionality to support the composition and delivery of personalized healthcare educational programs.

Cardiovascular diseases (CVD) place a significant burden on health professionals, patients and their care-givers, and extract significant health care costs. Thus, there are important gains in its prevention that can be achieved through risk factor modification and healthy lifestyle changes [4]. Risk factor modification is addressed through patient education to empower patients to self-manage disease risk and improve their quality of life [2]. However, it is argued that educational interventions may not necessarily have the desired impact if they do not take into account the patient's behavioural attitudes towards self-management and self-improvement. Personalization of educational interventions, therefore, should not only account for the patient's current health profile but in addition the patient's perceptions to health 


\section{Selena Davis and Syed Sibte Raza Abidi}

and readiness to change. We hypothesize that the above elements can realize objective user profiles and lead to the provision of personalized web-mediated interventions in terms of interest, knowledge, and compliance to the suggested lifestyle modifications of patients.

In this paper, we present our approach and proposed system - PULSE (Personalization Using Linkages of SCORE and behaviour change readiness to webbased Education). Our approach is grounded in the observation that the efficacy of any patient educational intervention is contingent on the patient's behaviour change readiness. As such, we use both CVD risk assessment and behavioural change readiness tool-in particular the Transtheoretical Model (TTM) - to determine the patient's profile; based on the profile, our personalization algorithm selects relevant messages to compose the educational material for an individual patient. We use Systematic COronary Risk Evaluation for risk assessment and Stages of Change for behaviour change readiness assessment. The educational material is derived from ProChange Behavior Systems Inc. and Canadian clinical guidelines. The personalization decision logic is represented in terms of Medical Logic Modules (MLM), implemented in Java. Finally, a web-based adaptive hypermedia system is being developed to generate and deliver the personalized education material to the patient.

\section{Information Personalization for Healthcare}

Patient education involves a set of planned, educational activities designed to improve patients' health behaviours and/or health status [5]. Personalization of healthcare information with respect to the patient's characteristics and health needs is an increasing trend as patients are taking an active interest and charge of their healthcare needs. The literature suggests that patients want: more information about their illness and treatment plan than they typically receive during physician visits; information that is custom-tailored to their own situation; information when they formulate questions about their health issues, which is generally after leaving the clinic and not during the physician visit; information that is endorsed by their physician as credible and applicable to their specific problem; information from other sources, such as journal articles and websites; and, information that they can retain for future reference [6].

Computer-tailored education programs, using adaptive hypermedia methods, generate comprehensive assessments of health-related behaviours at an individual level and compose personalized healthcare information by adapting the message, source of information, and/or the method of delivery. According to Jones et al., [7]computer-based information personalization requires at least five components, including: 1) a user profile, 2) a digital library containing all messages, 3) a mapping schema that generates the appropriate messages, 4) a document template for appropriate allocation and display of messages, and 5) a medium to deliver the message to the intended user. Web dissemination of tailored health interventions has demonstrated positive impact on determinants of behaviour change [8].

In our work we have incorporated behavior change considerations within the information personalization framework. The rationale being that the impact of any personalized information can be maximized if it aligns with the patient's state of readiness to uptake the recommended information. Research and experience reveal 


\section{Adaptive Patient Education Framework Featuring Personalized Cardiovascular Risk Management Interventions}

that initiating and maintaining positive behaviour changes is challenging for most people. Prochaska's TTM of intentional behaviour change is a stage-based model founded on 25 years of research. The model matches the change principles and processes to each individual's current stage of change, in order to guide them through the process of modifying problem behaviours and acquiring positive behaviours [9].

The use of fully integrated TTM constructs to inform the design of personalized messages has been effective for intervening across a broad range of health-related behaviors [10]. More specifically, results in tobacco control studies with interventions tailored to a smoker's stage were successful more often than non-tailored interventions in promoting forward stage movement [11].

\section{PULSE: Design and Development}

\subsection{Patient Data Capture and Profile}

We use the validated, commercially available Wellsource Coronary Risk Profile as the basis of our Data Capture Template (DCT) for collecting patients' demographic, behavioural, and clinical risk factor characteristics. The global INTERHEART Study [12] indicates that the nine risk factors of smoking, lipids, hypertension, diabetes, obesity, diet, physical activity, alcohol consumption, and psychosocial factors account for over $90 \%$ of the risk of acute MI. These factors are captured in our DCT to design an objective patient profile.

We represent the patient profile in three parts as each component collects patient parameters for specific personalization purposes: (1) CVD Risk Profile determined through the SCORE algorithm that estimates the 10-yr total cardiovascular risk of death. Patient data on age, gender, smoking, systolic BP, and total cholesterol and HDL cholesterol ratio is used to calculate the patient's risk category [13]; (2) Staged Risk Factor Profile depicts the patient's Stage of Change for specific modifiable risk factor behaviours. The Staged Risk Factor Profile is determined by a patient's response to questions relating to her/his readiness to change modifiable risk factor behaviours - smoking, being overweight, stress, depression, and exercise; and (3) Non-staged Risk Factor Profile determined by risk factor values-i.e. diet, alcohol, LDL cholesterol, Triglycerides, diastolic BP, glycemic control, and personal and family health history.

\subsection{Message Library}

In the PULSE program, the educational interventions are specifically designed to address both the medical and psycho-social aspects of CVD risk management. As such, we use a combination of staged lifestyle modification materials and risk-specific messages based on clinical guidelines to provide a valid use of behaviour change theory and Canadian sources of clinical and lifestyle modification education.

The various sourced materials are broken down into small "snippets of information", <tagged>, and stored in an SQL database. The XML <tag> for each 


\section{Selena Davis and Syed Sibte Raza Abidi}

snippet follows an indexing schematic which provides mapping ease to the patient profile for personalization purposes (e.g. < smoking>).

\subsection{Decision Logic}

Given a patient profile and a message library containing an assortment of education interventions, the personalization mechanism involves the selection of the most relevant set of messages based on the patient's profile. We design a personalization matrix to summarize the patient parameters assessed and describe specific combinations of characteristics that lead to a certain message. Personalization is achieved through the processing of a set of symbolic rules based on decision logic that maps the profile elements to specific messages. We develop a rule-based inferencing engine that incorporates the decision logic. To represent our medical knowledge we use MLMs, a standard for independent units composing a series of rules in health knowledge bases. The entire decision logic, sets of MLMs, is implemented in Java and represented as a comprehensive decision tree describing each of the risk factors and risk conditions contained in the patient profile. Each MLM consists of four parts: an evoking event, logic, action, and data mapping. The logic contains "if-then" rules, where the IF part of the rules contains variables for the patient profile. Using case statements, if the IF part of the rule is not satisfied then the engine directs the execution of the next case statement. If the IF part of the rule is satisfied-i.e. the patient's profile matches the rule constraints then the rule fires and the THEN part of the rule becomes available for execution. Typically the THEN part contains a list of messages that are to be selected as the patient's personalized educational material.

\subsection{Presentation \& Delivery}

We pre-designed a hypermedia template for the output whereby relevant messages selected for an individual patient are structured and presented in a consistent manner. Such content adaptation ensures each patient's personalized document is coherent and contains only the information that is pertinent to the patient. A web-based delivery medium is used to deliver the information to the patients via the practitioners. A print version is available for patients without computer access.

\section{Concluding Remarks}

In this paper we have presented the preliminary design of our computer-tailored patient education strategy that features: (a) Usage of SCORE for risk assessment; (b) Incorporation of behaviour change inputs in determining the educational content as opposed to just relying on medical data; (c) Usage of an objective DCT currently operational; (d) Leveraging Canadian clinical guidelines for both deriving the decision logic and the corresponding educational intervention; and (e) Personalization of educational material. The realization for personalized education information compared to generic information has led to various computer-tailored healthcare 


\section{Adaptive Patient Education Framework Featuring Personalized Cardiovascular Risk Management Interventions}

educational programs [14]. The project is underway and we would be reporting detailed implementation and evaluation studies in a separate publication. Here, we state a case for the application of adaptive personalization methods for patient education programs; such programs can help reduce disease risks and deal with risk management by influencing patient behaviour changes through the provision of pertinent lifestyle modifications and change strategies.

\section{References}

[1] H.Q. Nguyen and V.C. Kohlman, "Internet-based patient education and support interventions: A review of evaluation studies and directions for future research," Computers in Biology and Medicine, vol. 34, pp. 95-112, 2004.

[2] T. Hoffmann, T. Russell and K. McKenna, "Producing computer-generated tailored written information for stroke patients and their carers: system development and preliminary evaluation," International Journal of Medical Informatics, vol. 73, pp. 751-758, 2004.

[3] J. Brug, M. Campbell, and P. van Assema, "The application and impact of computer-generated personalized nutrition education: A review of the literature," Patient Education and Counseling, vol. 36, pp. 145-156, 1999.

[4] D.G. Manuel, M. Leung, K. Nguyen, P. Tanuseputro and H. Johansen, "Burden of cardiovascular disease in Canada," Canadian Journal of Cardiology, vol. 19, pp. 997-1004, 2003.

[5] K. Lorig, T.R. Prohaska et al., Patient education: a practical approach. Sage Publications, 2001.

[6] M.W. Kreuter and R.J. Wray, "Tailored and targeted health communication: strategies for enhancing information relevance," American Journal of Health Behaviour, vol. 27, pp. S227-S232, 2003.

[7] B. Jones, S.S.R. Abidi and W. Ying, "Using Computerized Clinical Practice Guidelines to Generate Tailored Patient Education Materials," in 38th Hawaii IEEE International Conference on System Sciences, 2005, pp. 139b.

[8] A. Oenema, J. Brug and L. Lechner, " Web-based tailored nutrition education: results of a randomized controlled trial," Health Education Research, vol. 16, pp. 647-660, 2001.

[9] Cancer Prevention Research Center, "Detailed Overview of the Transtheoretical Model," December 15, 2004. Available: http://www.uri.edu/research/cprc/TTM/detailedoverview.htm

[10] J.A. Sarkin, S.S. Johnson, J.O. Prochaska and J.M. Prochaska, "Applying the Transtheoretical Model to Regular Moderate Exercise in an Overweight Population: Validation of a Stages of Change Measure,” Preventive Medicine, vol. 33, pp. 462-469, 2001.

[11] L. Spencer, F. Pagell, M.E. Hallion and T.B. Adams, "Applying the transtheoretical model to tobacco cessation and prevention: a review of literature," American Journal of Health Promotion, vol. 17, pp. 7-71, Sept-Oct. 2002

[12] S. Yusuf, S. Hawken, S. Ôunpuu, T. Dans, A. Avezum, F. Lanas, M. McQueen, A. Budaj, P. Pais and J. Varigos, "Effect of potentially modifiable risk factors associated with myocardial infarction in 52 countries (the INTERHEART study): case-control study," The Lancet, vol. 364, pp. 937-952, 2004.

[13] R.M. Conroy et al., "Estimation of ten-year risk of fatal cardiovascular disease in Europe: the SCORE project," European Heart Journal, vol. 24, pp. 987-1003, 2003.

[14] U.S. National Institutes of Health, "Trial of a Tailored Message Program to Implement CHF Guidelines," Jan. 6, 2006. http://www.webcitation.org/1143495385123127 [accessed 2006 Jan 9] 